\title{
Effects of Processing and Composition on the Structure Formation in Casein Based Food Systems
}

\author{
Sonja Röck and Ulrich Kulozik
}

Chair for Food Engineering and Dairy Technology, Technische Universität München, D-85354 Freising-Weihenstephan. E-mail: Sonja.roeck@wzw.tum.de

A multistage structure formation process is observed in systems such as fresh cheese or processed cheese. It is unclear so far which reactions control the structure formation process and how processing and compositional factors influence the structure formation. Therefore, the objective of this study is to clarify which mechanisms are responsible for the structure build-up. These findings are used to develop an indepth understanding of these complex reactions.

The investigations are carried out with a rheometer to track the structure formation course online during the processing. It was found that the structure formation reaction follows a typical time course through four different phases (lag -, exponential I -, plateau - and exponential phase II). The shape of the viscosity curve can be influenced by processing and compositional factors. The following processing factors were investigated: shear intensity, temperature and an upstream homogenisation of the fat phase. The experiments showed that higher shear intensities result in an acceleration of the structure formation process. This can be explained by the intense mixing of the ingredients leading to an increasing contact probability of the proteins. Therefore, the creation of a protein network can take place earlier. Furthermore, it was assessed that a processing temperature of $70{ }^{\circ} \mathrm{C}$ and higher is necessary to induce the structure formation. An upstream homogenisation of the fat phase, which influenced the incoming droplet size of the fat globules, leads to a faster reaction.

Regarding composition, the kind and content of protein, fat level and $\mathrm{pH}$ were varied. The experiments showed that the presence of fat is indispensable, without fat the reaction hardly takes place. A minimum fat level of $15 \%$ is required to induce the reaction. Light micrographs highlight the important role of fat for the structure formation process demonstrating that the fat droplets size decreased with increasing processing time. As shown in figure 1, an increasing protein content speeds the reaction up. By means of electron micrographs it was shown that the proteins migrate from the interface between fat and protein to concurrently generate a protein network in the continuous phase. In summary, two reactions seem to play a role in the structure formation process: A change in the state of emulsion and the formation of a protein network in the continuous phase.

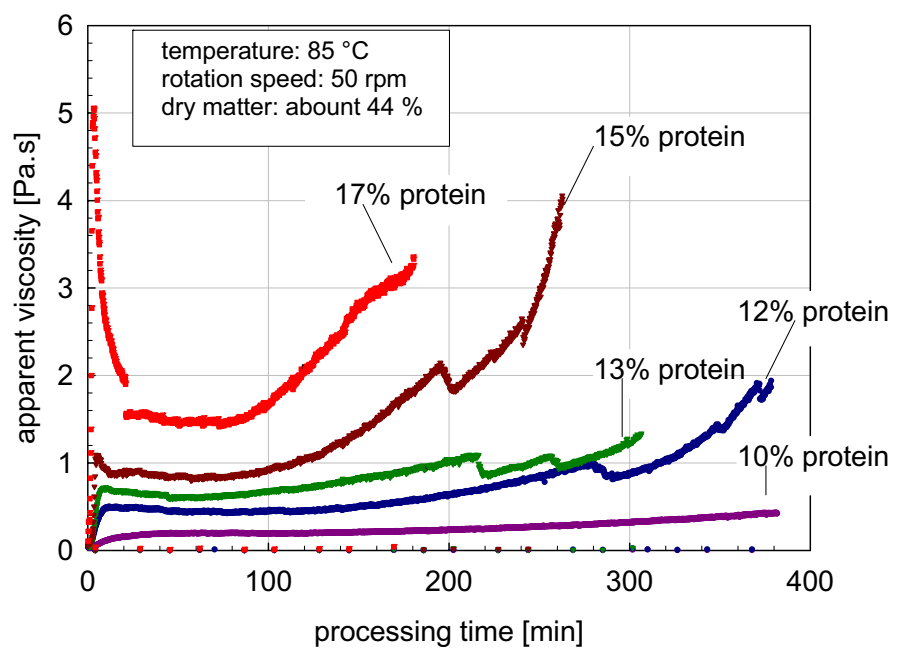

Fig. 1: Course of viscosity as function of processing time depending on the protein content in a dairy protein model system 